Bull. Mater. Sci., Vol. 6, No. 3, July 1984, pp. 537-547. Printed in India.

\title{
Electron microscopy of defect clusters produced by radiation damage
}

\author{
S BANERJEE \\ Physical Metallurgy Division, Bhabha Atomic Research Centre, Bombay 400085, India
}

\begin{abstract}
The methods of characterization of different types of radiation-induced defect clusters by TEM have been reviewed. Point defects produced in irradiated materials agglomerate in two or three dimensional clusters to reduce the strain energy associated with them. Two-dimensional clusters assume the configuration of vacancy or interstitial type dislocation loops which can be resolved if the size of the loops is large compared to the extinction distance associated with the imaging reflection. The small loops give rise to a black dot contrast under the kinematical and a black-white contrast under the dynamical imaging conditions. The method of characterization of dislocation loops which include the determination of the nature of the loop, the Burgers vector and the loop plane normal is discussed taking examples from the work done on the ion irradiated $\mathrm{Ni}_{4} \mathrm{Mo}$ samples. A summary of available experimental results on the characterization of dislocation loops in different metals and alloys having fcc, bcc and hcp structures is presented. The contrast from stacking fault tetrahedra which form in some foc metals and alloys after a certain degree of annealing is also discussed. The optimum conditions for imaging three-dimensional clusters or voids are derived on the basis of the contrast theory proposed for such defects. Special reference is made to the usefulness of "through focus analysis" in the imaging of very small cavities (with diameters as small as about $10 \AA$ ).

It is shown that the formation of disordered zones resulting from displacement cascades in the ordered matrix can be utilized in determining the shape and the volume of cascades in the virgin state. The importance of different contributing factors like the strain contrast and the structure factor contrast in producing the overall contrast from the disordered zones is discussed. Detailed observations on the shape of the disordered zones are shown to be important to establish the occurrence of the replacement collision sequence and the formation of sub-cascades.
\end{abstract}

Keywords. Radiation damage; electron microscopy; point defect clusters; disordered zones.

\section{Introduction}

Energetic particle irradiation of metals causes permanent displacement of atoms if the recoil energy transferred from the incident particles to the target atoms exceeds the displacement energy. Depending on the momentum and the energy, an incident particle can produce either a Frenkel type defect consisting of a single vacancy-interstitial pair or a cascade of displacements. The latter process results in creating a depleted zone having a vacancy-rich core surrounded by a region with a high density of interstitials. Such a depleted zone structure is not stable due to the high strain energy associated with it. A substantial part of the strain energy is released when vacancies in the core of the depleted zone collapse into a two dimensional cluster assuming the configuration of a vacancy type dislocation loop. When irradiation causes production of isolated point defects, their concentrations build up and depending on the extent of supersaturation, they tend to cluster as point defect agglomerates (PDA). Isolated point defects are not observable in TEM at the present time, but PDAS which assume configurations like dislocation loops, stacking fault tetrahedra, cavities etc give rise to specific contrast 
effects on TEM images. The present paper deals with the methods of characterization of PDAs by studying the details of their contrast.

As mentioned earlier, the formation of a dislocation loop at the core of a cascade involves migration of point defects and also annihilation of defects through the vacancy-interstitial recombination. As a result, dislocation loops do not truly represent the cascades associated with them in their virgin state. A better imaging of cascades has been possible in ordered alloys in which the cascade volumes are disordered by incident particles. This novel technique of imaging cascades will also be discussed here.

\section{Contrast from small dislocation loops}

Earlier investigations have shown that radiation damage in metals causes formation of defects, which appear as black dots of irregular shape when the sample is imaged in the kinematic bright field condition i.e. when no low order reflection is strongly excited. It was not possible to derive any crystallographic information regarding the nature and the geometry of the radiation induced defects from such black dot contrast. Essman and Wilkens (1964), for the first time, detected that the black dots in neutron irradiated copper revealed a characteristic black-white contrast when the samples were imaged under two-beam dynamical contrast condition (with excitation error, $s \approx 0$ ). From a systematic study of such black-white contrast, both theoretical and experimental, the methods of finding out various crystallographic parameters such as the nature of the loop, the Burgers vector, the loop plane normal associated with a defect cluster have now been established mainly from the work carried out by Wilkens and coworkers in Stuttgart and Eyre and his coworkers in Harwell. This subject has been dealt with extensively by Eyre (1972), Ruhle (1967a,b) and Wilkens (1970, 1973).

Some of the theoretical results based on which defect analysis can be done are summarised as follows: (i) Only those defects which lie within a distance of about 1.5 times $\xi_{g}$ from the top and the bottom surfaces of the foil give rise to the black-white contrast under the two beam dynamical imaging condition with $\mathbf{g} \cdot \mathbf{b} \neq 0$ where $\mathbf{g}$ is the operating reflection and $\mathbf{b}$ the Burgers vector. The disappearance of the black-white contrast from small loops located at distances exceeding $1.5 \xi_{g}$ is due to anomalous absorption. The factor $\xi_{g}^{\prime} / \xi_{g}$ where $\xi_{g}^{\prime}$ is the anomalous absorption length provides a guide line for determining the maximum depth up to which a defect produces a blackwhite contrast. (ii) For loops in the middle region of the foil, i.e. outside the region of observable black-white contrast, a black dot contrast is expected. (iii) As mentioned earlier, dislocation loops located near the surfaces of the foil reveal a black-white contrast when $\mathbf{g} \cdot \mathbf{b} \neq 0$ and $s \approx 0$. If a vector $\mathbf{l}$ is so defined that it points from the centre of the black lobe to the centre of the white lobe of the contrast figure in the positive print, then the sign of $\mathrm{g} \cdot \mathbf{l}$ oscillates with depth as shown schematically for vacancy loops in figure 1. The orientation of 1 remains independent of the direction of $g$ and lies parallel to $\mathbf{b}$ or its projection on the image plane. This statement is, however, valid only for angles between $\mathbf{g}$ and $\mathbf{b}<55$ to $60^{\circ}$ (Ruhle $1967 \mathrm{a}, \mathrm{b}$ ). Image simulation results for the case of $\frac{1}{2}\langle 110\rangle$ and $\frac{1}{2}\langle 111\rangle$ edge loops in a boc crystal show that I deviates from $+b$ (or its projected direction) by an amount that increases as $\alpha$, the angle between $\mathbf{b}$ and $\mathbf{g}$ (Eyre 1972). (iv) Eyre (1972) has shown that for fairly large values of $\mathbf{g} \cdot \mathbf{b}(\gtrsim 4 / 3)$ a fine structure is developed at the separation line between the black and the white lobes. (v) When $\mathbf{g} \cdot \mathbf{b}=0$, a contrast figure often described as a butterfly contrast (Wilkens et al 
D. F. B. $F$

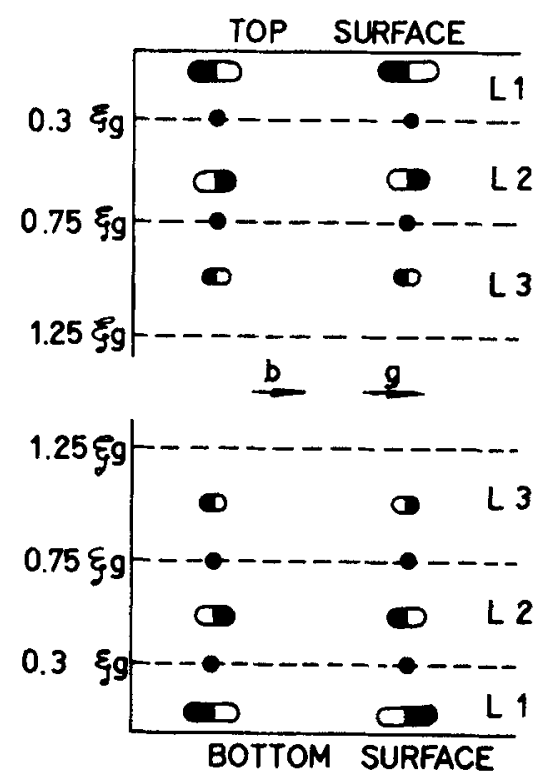

Figure 1. Schematic illustration, of the black-white contrast from small dislocation loops of vacancy type located at different depths of the sample. Bright (BF) and dark field (DF) image characteristics as seen on positive prints. For interstitial type loops the same image characteristics are seen for $\mathbf{g}$ pointing in the reverse direction.

1970) which contains three black and three white lobes is observed. (vi) Under the kinematical imaging condition i.e. for large excitation errors $\left(|s| \cdot \xi_{g} \gg 1\right)$ only black dot contrast remains for all loop positions. Since the diameter of the black dot contrast, measured for instance at $20 \%$ image width, closely corresponds (within about $10 \%$ error) to the projected loop width, such kinematical images are very useful in the quantitative determination of the loop size.

Based on the aforementioned principles of image formation, it is possible to identify the nature of a small dislocation loop (i.e. either vacancy or the interstitial type) and determine the Burgers vector and the loop plane normal. The method is illustrated taking examples from a recent experimental work on ion irradiation induced damage in $\mathrm{Ni}_{4}$ Mo. (Banerjee et al (unpublished)).

\subsection{The sign of the misfit volume}

The vacancy and the interstitial type loops with misfit volumes, $\Delta V<0$ and $\Delta V>0$ respectively can be distinguished from the sign of $\mathbf{g} \cdot \mathbf{l}$ provided the depth position of the defect is determined accurately by the stereomicroscopic method. In $\mathrm{Au}^{++}$ion irradiation on $\mathrm{Ni}_{4} \mathrm{Mo}$, the range of the incident ion was such that almost all defects were formed within the layer, L1 (refer figure 1). Therefore, in the present case, it was not necessary to find out the depth positions of these defects. Comparing figures 1 and 2 it can be seen that these defects are all vacancy loops, that l vectors for different loops are pointing along different directions and all are certainly not along the $g$ vector. This observation clearly demonstrates that the strain field around these defects are not 


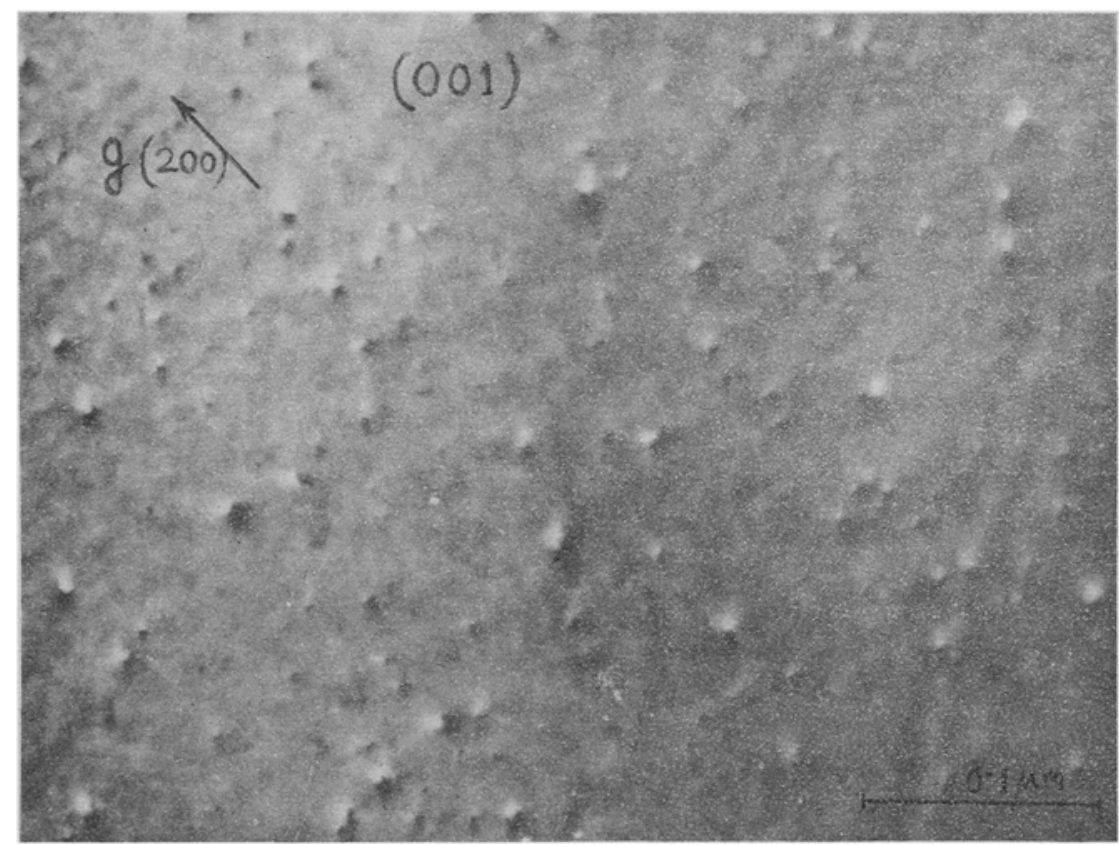

Figure 2. Black-white contrast from vacancy type dislocation loops produced in $\mathrm{Ni}_{4} \mathrm{Mo}$ irradiated with $60 \mathrm{keV} \mathrm{Au}{ }^{++}$ions. Note that all 1 vectors are not parallel to $\mathrm{g}$.

spherically symmetric because in that case the I vector is expected to be always parallel to g. Analysis of contrast figures of defect clusters in $\mathrm{Nb}_{3} \mathrm{Sn}$ (Jenkins et al 1982) has shown such contrast character and they suggested the formation of amorphous disordered zones in the ordered matrix which results in the production of spherically symmetric strain centres.

\subsection{Burgers vector and plane normal $\mathbf{n}$ of a small dislocation loop}

The method of determining the Burgers vector $\mathbf{b}$ and the loop plane normal of a resolvable dislocation loop involves finding out a suitable $g$ for which contrast extinction occurs and tilting experiments to bring the loop plane parallel to the electron beam direction. For the unresolved loops, a careful comparison of the contrast figure from a given set of dislocation loops under different imaging reflections enables one to determine these parameters unambiguously. Strictly speaking, a comparison between computer simulated images of dislocation loops with different $\mathbf{b}$ and $\mathbf{n}$ under various conditions of imaging ( $\mathrm{g}$ and foil plane normal) is necessary. The analysis, however, turns out to be simpler in many cases where possible Burgers vectors are restricted by crystallographic arguments. Figure 3 illustrates one such example where loops are grouped into three types $\mathrm{A}, \mathrm{B}$ and $\mathrm{C}$ depending on the contrast figures exhibited by them under different $g$ all on the same foil plane $(001)$. The l vectors are seen to be along $A, B$ and $C$ vectors which are along projections of different $\langle 110\rangle$ and $\langle 111\rangle$ directions. Table 1 shows that if $\mathbf{b}$ for $\mathrm{A}, \mathrm{B}$ and $\mathrm{C}$ types of dislocation loops are taken to be those indicated in the last column of the table 1, the observed contrast figure 

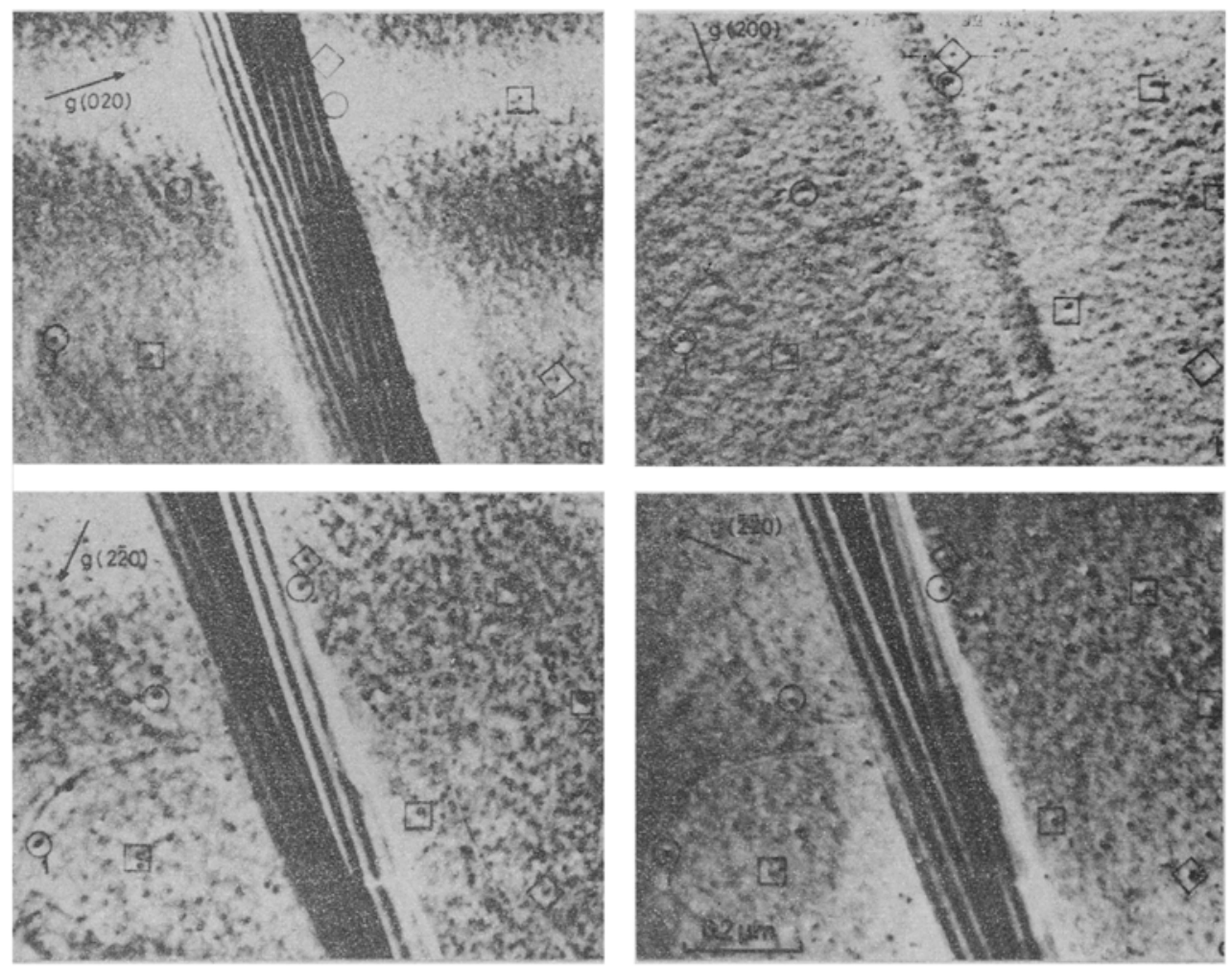

Figure 3. Analysis of Burgers vector of small dislocation loops. Based on the contrast character (as listed in table 1) under different operating reflections loops are grouped into three types $\mathrm{A}, \mathrm{B}$ and $\mathrm{C}$ marked by circle, square and diamond markers.

matches with what is expected from theoretical considerations. Based on this observation, it has been concluded that apart from the Frank loops, some perfect loops are also formed in the ion irradiated ordered $\mathrm{Ni}_{4} \mathrm{Mo}$ alloy. Figure 4 illustrates the details of the contrast figure of perfect and Frank loops under different imaging conditions with increasing magnitude of $\mathbf{g}$. $\mathbf{b}$. The internal contrast at the centre of the black-white figure is clearly visible when $\mathbf{g}$. $\mathbf{b}$ is close to 2 .

Experimental work on several fcc metals has established that a great majority of small dislocation loops are of the Frank type which have pure edge character. The rule that the $\mathbf{l}$ vector is parallel to $\mathbf{b}$ or the projection of $\mathbf{b}$ is valid in such cases and therefore unambiguous identification of these loops is possible using this property of the loop contrast. However, this property alone is not sufficient to determine $\mathbf{b}$ and $\mathbf{n}$, particularly for shear loops.

In bcc metals, Eyre and Bullough (1965) have shown from theoretical consideration that small loops are nucleated on $\{110\}$ planes and in a subsequent shear process the Burgers vector $\mathbf{b}=\frac{1}{2}\langle 110\rangle$ is converted into $\mathbf{b}=\frac{1}{2}\langle 111\rangle$ or, less probably, into $\mathrm{b}=\langle 100\rangle$. Experimental work on ion irradiated Mo and on $\mathrm{W}$ (Eyre et al 1977) have shown that a majority of the loops in bcc metals have $\mathbf{b}=\frac{1}{2}\langle 111\rangle$ and $\mathbf{n}=\langle 110\rangle$. No evidence for loop with $\mathbf{b}=\langle 100\rangle$ was found in $\mathrm{W}$.

In Co (Wilkens 1978) nearly all loops could be indexed with $\mathbf{b}=1 / 3\langle 1 \overline{120}\rangle$ and $\mathbf{n}=\langle 10 \overline{010}\rangle$. No radiation induced loops on the basal plane could be detected. 
Table 1. Image characteristics of loops shown in figure 3.

\begin{tabular}{|c|c|c|c|c|c|c|}
\hline \multirow{2}{*}{$\begin{array}{l}\text { Type } \\
\text { (Marker) }\end{array}$} & & \multicolumn{4}{|c|}{ g } & \multirow[t]{2}{*}{ b } \\
\hline & & {$[020]$} & {$[200]$} & [220] & {$[2 \overline{2} 0]$} & \\
\hline \multirow{3}{*}{$\begin{array}{l}\text { A } \\
\text { (Circle) }\end{array}$} & 1 & & $\mathbf{A}$ & $\mathbf{A}$ & $\mathbf{A}$ & \\
\hline & shape & butterfly & BW & BW & BW & $\frac{1}{2}[101]$ \\
\hline & $\mathbf{g} \cdot \mathbf{b}$ & 0 & 1 & 1 & 1 & $\frac{1}{2}[10 \overline{1}]$ \\
\hline B & $\begin{array}{c}1 \\
\text { shape }\end{array}$ & $\begin{array}{c}\text { B } \\
\text { BW }\end{array}$ & $\begin{array}{c}\text { B } \\
\text { BW }\end{array}$ & butterfly & $\begin{array}{c}\text { B } \\
\text { BW }\end{array}$ & $\begin{array}{l}1 / 3[1 \overline{11}] \\
\text { or }\end{array}$ \\
\hline (square) & $\mathbf{g} \cdot \mathbf{b}$ & $2 / 3$ & $2 / 3$ & 0 & $4 / 3$ & $1 / 3[1 \overline{11}]$ \\
\hline C & $\begin{array}{c}1 \\
\text { shape }\end{array}$ & $\underset{B W}{C}$ & $\underset{B W}{C}$ & $\begin{array}{l}\text { C } \\
\text { Is }\end{array}$ & butterfly & $\frac{1}{2}[110]$ \\
\hline (Diamond) & $\mathbf{g} \cdot \mathbf{b}$ & 1 & 1 & 2 & 0 & \\
\hline
\end{tabular}

A parallel to projection of [101] and [101];

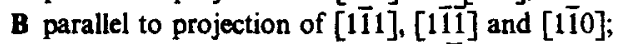

C parallel to projection of [111], [111] and [110]. B W represents blackwhite contrast with no prominent internal structure. I $S$ represents black white contrast with prominent internal structure.

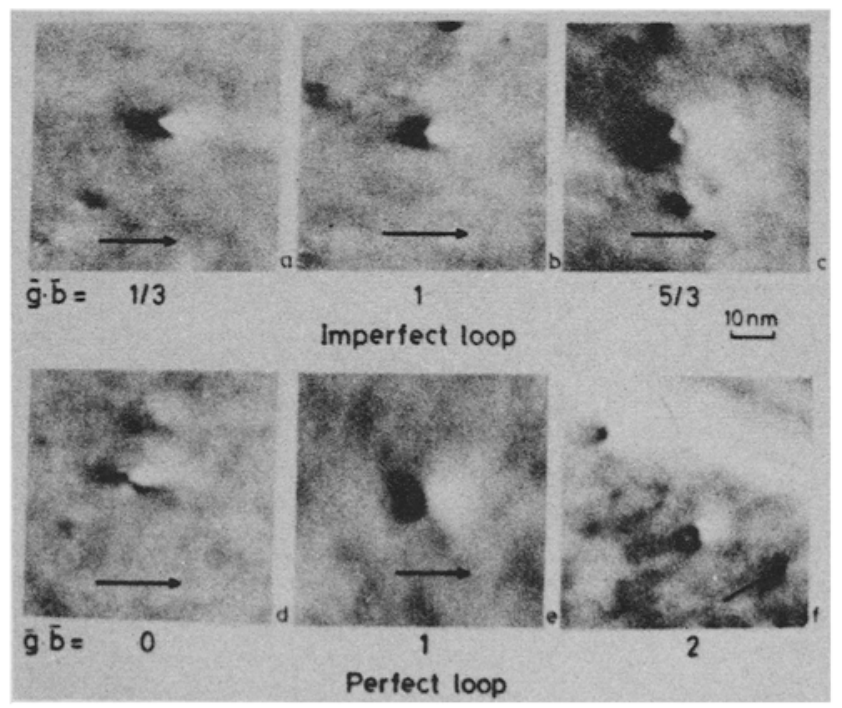

Figure 4. The influence of the magnitude of $g \cdot b$ on the details of the loop contrast.

\section{Contrast from voids}

Theoretical contrast calculations have been carried out by many investigators and on the basis of these results, Eyre (1972) has listed the Following image characteristics for voids: 
(i) Voids give rise to strain contrast absorption contrast and phase contrast and relative magnitude of each of these very strongly depends on the void size, the thickness of the sample and the imaging condition. (ii) In a two-beam dynamical image, the largest contribution to in-focus images comes from absorption contrast except for very small cavities which produces strain contrast to a significant extent. (iii) When absorption contrast is predominant, contrast is dictated by foil thickness. The optimum contrast is obtained in a narrow region at the front of the first and second dark thickness fringe (figure 5). It has been demonstrated that cavities exhibit positive contrast at the bright and negative contrast at the rear or dark fringes in bright field, the reverse being true in the dark field. (iv) In thicker foils, anomalous absorption damps out the oscillation in intensity and all cavities exhibit above background contrast, due to a change in the normal absorption. (v) The contrast from very small voids can be obtained only in out of focus imaging, i.e. when objective lens is focussed on a plane at a distance, $\zeta$ (about $1 \mu \mathrm{m}$ ) from the bottom layer of the specimen. By this technique, cavities with diameters $10 \AA$ or less can be made visible. Ruhle (1971) have shown that cavities degenerate with increase in thickness into pure phase objects in the electron optical sense. Such phase objects are invisible under in-focus conditions. The phase shift is given by $\Delta V$, the difference between the mean inner potentials inside the cavity and in the crystalline matrix. The contrast figure obtained in the defocus mode consists of a central region which in bright or dark depending on the sign of $\Delta V$ and $\zeta$ and a number of Fresnel fringes of alternating sign surrounding the central region. 'Through focus imaging' technique can also be used, in principle, to establish $\Delta V$ because the contrast depends on the sign and the magnitude of $\Delta V$ for cavities which are large enough to be detectable under the in-focus imaging condition.

\section{Contrast from disordered zones}

In pure metals and disordered alloys the size of the vacancy loops produced by the collapse of vacancies at the core of displacement cascades gives a measure of the cascade size. The loops, however, do not truly represent the cascades because only the vacancies which survive in a cascade core make up a dislocation loop and vacancies undergo a considerable degree of rearrangement during the collapsing process. When displacement cascades are produced in ordered alloys, random displacements and replacements of atoms destroy the order within the cascade. Dark field image taken with a strongly excited superlattice reflection then shows a distribution of disordered zones embedded in the ordered matrix. The contrast arises primarily due to the difference in the structure factor for the superlattice reflections between the disordered zone and the ordered matrix. When such disordered zones are associated with dislocation loops, additional contribution from strain contrast also appears. Different factors which influence the image contrast of disordered zones have been evaluated by Jenkins et al $(1976 \mathrm{a}, \mathrm{b})$ both theoretically and experimentally on the $\mathrm{Cu}_{3} \mathrm{Au}$ alloy. More recently, experiments on disordered zones in $\mathrm{Ni}_{4} \mathrm{Mo}$ (Banerjee et al unpublished) have confirmed earlier findings. The results of these investigations can be summarized as follows:

(i) The contrast was a sensitive function of foil thickness. Figure 6 shows the intensity relative to the background $\left(I / I_{0}\right)$ is foil thickness for disordered zones in $\mathrm{Ni}_{4}$ Mo imaged under 1/5 (420) and 3/5 (420) reflections (Banerjee et al unpublished). For the $1 / 5(420)$ superlattice reflection, the image intensity of the disordered zone is below the 


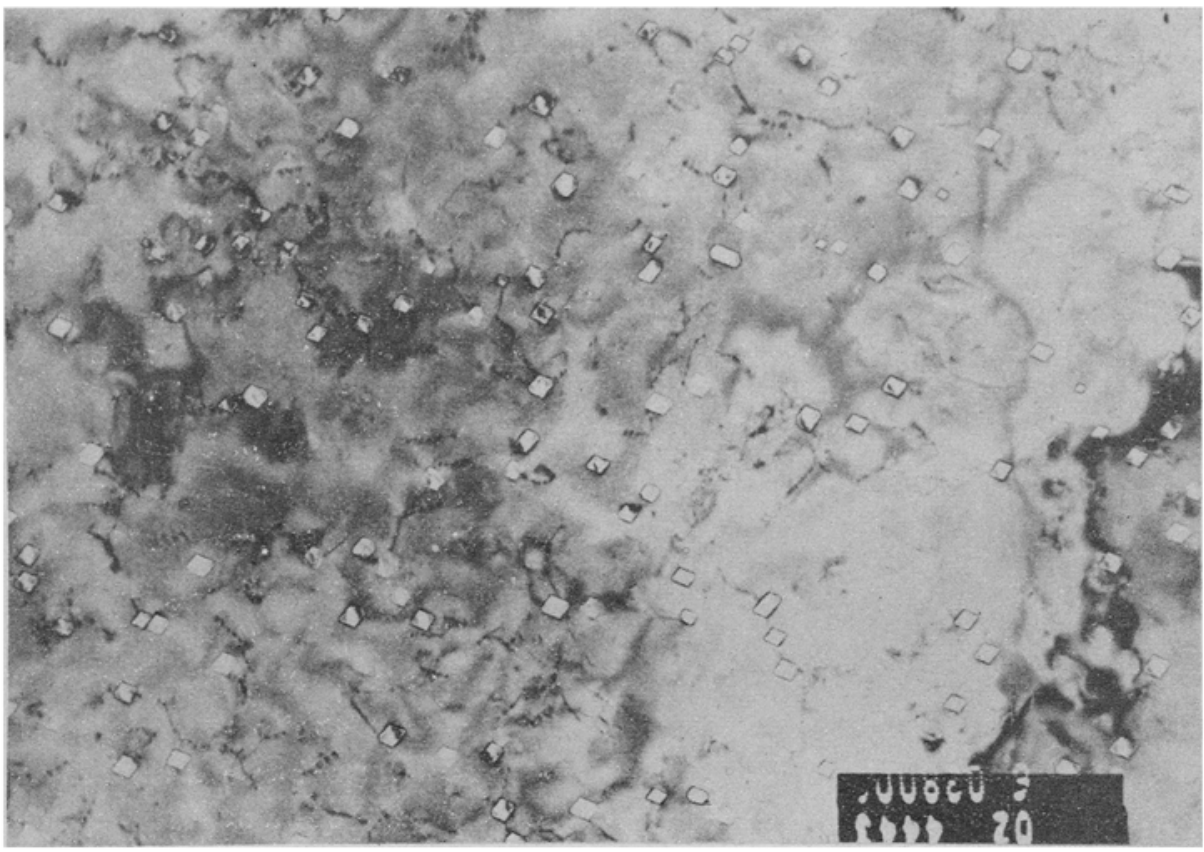

Figure 5. Contrast from voids in 316 stainless steel produced by $\mathrm{MeV}$ proton irradiation (courtesy S K Khera)

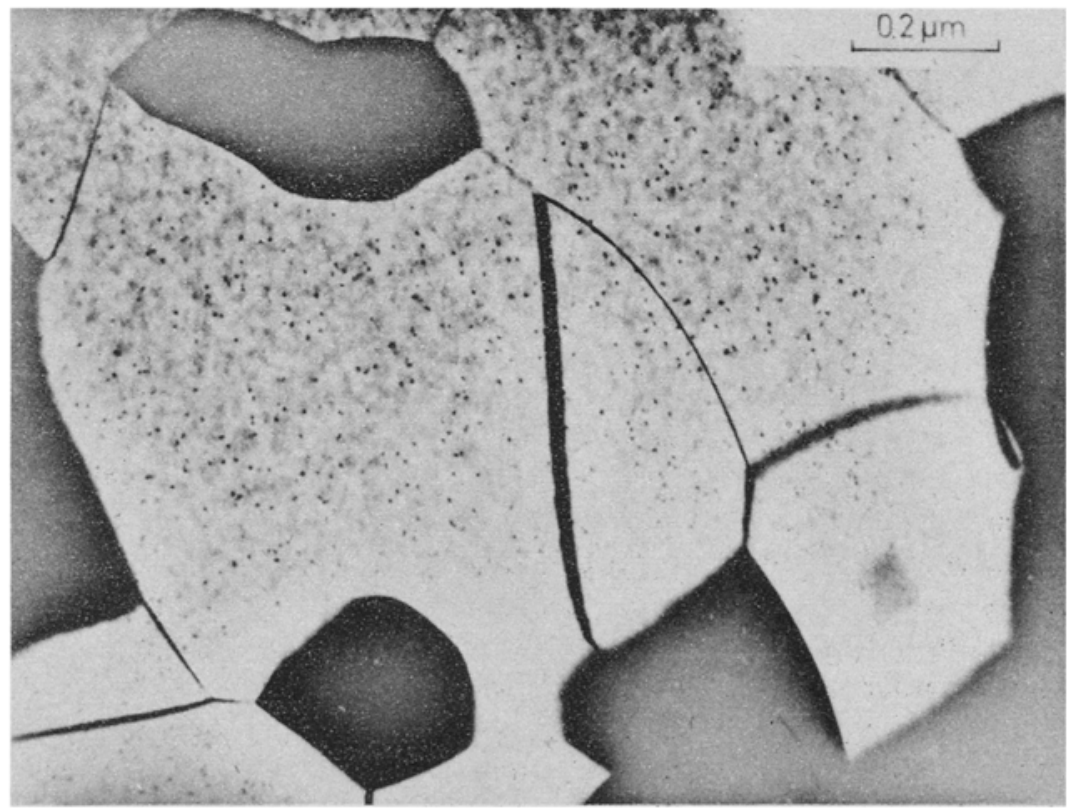

Figure 6. The calculated superlattice dark field contrast from a disordered zone of $5 \mathrm{~nm}$ in the matrix of the fully ordered $\mathrm{Ni}_{4} \mathrm{Mo}$. The influence of the degree of order(S) within the disordered zone and the specimen thickness on the contrast is shown. Calculation is based on twelve beams in a systematic row of reflections along $\langle 420\rangle$ real vectors with $g=1 / 5\langle 420\rangle$. 
background when the sample thickness is less than $850 \AA$ while for the $3 / 5(420)$ reflection the image intensity remains below the background for practically all electron transparent samples. The contrast, however, becomes very feeble as the sample thickness exceeds about $500 \AA$ and therefore disordered zones do not remain visible in thick regions of the sample. Figure 7 illustrates the contrast observed from disordered zones in $\mathrm{Ni}_{4} \mathrm{Mo}$ imaged under $1 / 5$ (420) reflection. (ii) The thickness of the disordered zone is another factor which strongly influences the contrast. Numerical calculations (Jenkins et al 1976 a) have shown that the contrast falls below the $10 \%$ level, commonly accepted as the visibility limit, for the disordered zone thickness smaller than about $20 \AA$. (iii) The contrast has been found to be linearly dependent on the long range order parameter, $S$ within the disordered zone. (iv) The contrast has been shown to be fairly insensitive to the extent of deviation from the exact Bragg orientation and also to the depth at which the disordered zone is located in a sample. It may be noted that the superlattice dark field contrast from disordered zones located at depth $Z_{0}$ is the same as that for disordered zones located at depth $\left(t-Z_{0}\right)$ where $t$ is the foil thickness. (v) Based on the calculations made by Jenkins et al (1976 a,b) in which contributions from both structure factor and strain constant are taken into account, it can be stated that the total contrast is dominated by the structure factor contribution for small disordered zones associated with dislocation loops (typically the loop diameter being less than about $40 \AA$ for $\mathrm{Cu}_{3} \mathrm{Au}$ imaged under (110) reflection). Even for larger disordered zones the size and the shape of the total contrast are fairly well represented by the contrast arising out of the structure factor contribution alone.

Because of the faithful reproduction of the shape of a cascade in the superlattice dark field image of the disordered zone associated with it, the details of the contrast figure

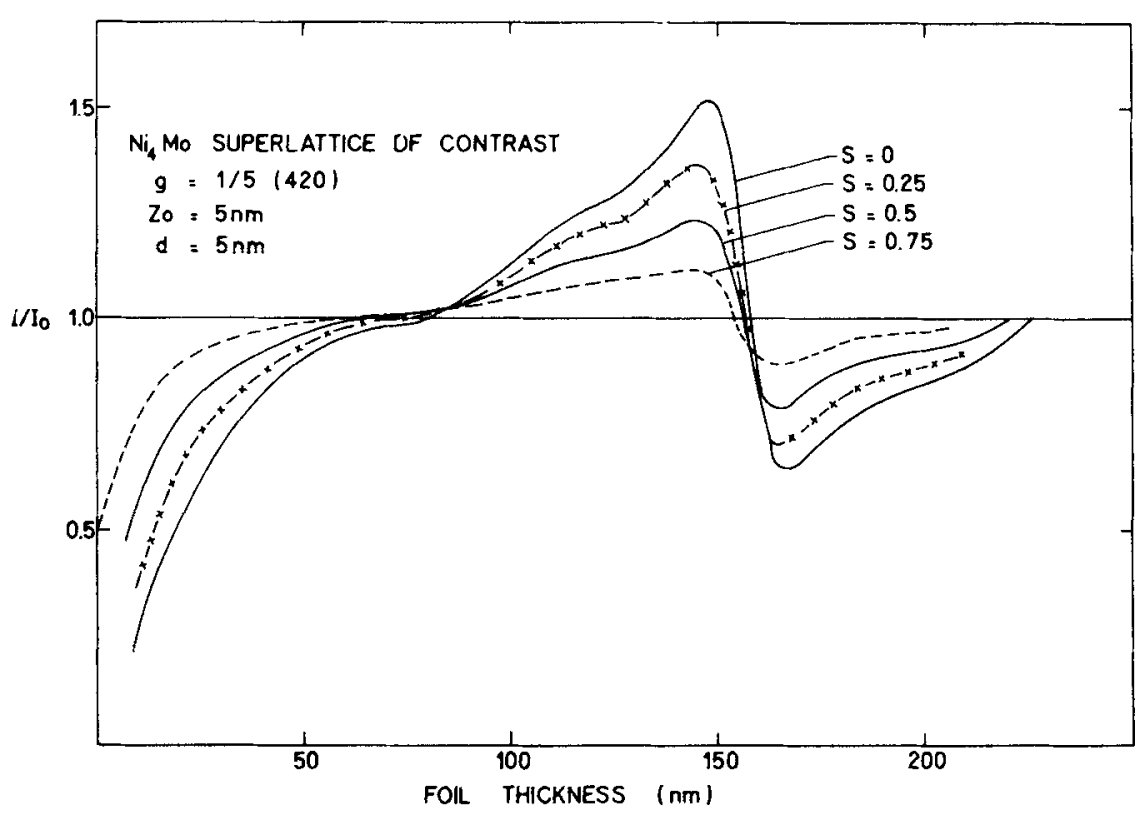

Figure 7. Dark field image with $1 / 5\langle 420\rangle$ reflection to show disordered zones in the matrix of ordered $\mathrm{Ni}_{4} \mathrm{Mo}$ irradiated with $60 \mathrm{KeV} \mathrm{Au}{ }^{++}$ions. Dark grains correspond to ordered domains related with bright domains by anti parallel twin relation. 
have been studied to explore the mechanism of displacement and replacement of atoms within the cascade volume. From the measurement of size of the cascades and assigning a distribution of disorder within the cascades the number of replacements, $n_{r}$ within a single cascade required to introduce the disorder can be computed. When this number is compared with the estimated number of atomic displacements, $n_{d}(\simeq E / 2 E d$, where $E$ is the energy of the incident ion and $E_{d}$ is an average displacement energy), one obtains $n_{r} / n_{d}$ to be in the range of 10 to 20 . This suggests that disordering is caused largely due to the replacement process. It has been envisaged that disordering may occur by the following processes to different extents: (i) formation of thermal spikes (ii) collapse of vacancies to form vacancy loops, and (iii) replacement collision sequence (RCS). To assess the role of RCS, detailed observation on the morphology of disordered zones has been made by Jenkins et al (1976b) and they have reported splitting up of disordered zones into two or more distinct dots and extrusions of the disordered zone into the matrix. Attempt has been made to examine whether these extrusions coincide with the close packed $\langle 110\rangle$ directions along which focussed RCs is expected to occur. But the results are not convincing enough to be certain that these extrusions are the path along which RCs has occurred. However, such a mechanism of propagation of RCs to produce sub-cascades in case of high incident ion energies $(>30 \mathrm{keV})$ still appears very probable.

\section{Concluding remarks}

At present TEM methods are well developed for complete crystallographic characterization of point defect agglomerates produced by radiation damage. The lattice resolution technique has not yet found any significant application in analysing these defect structures. With the availability of high resolution TEMs and growing interest in lattice resolution microscopy one may forsee some suitable applications of lattice resolution microscopy in this area in the near future.

\section{Acknowledgements}

The author is indebted to Dr M Wilkens for many helpful discussions. The unpublished results included in this review were obtained during the author's stay at the MaxPlanck-Institut fur Metallforschung as an Alexander-von-Humboldt fellow.

\section{References}

Banerjee S, Katerbau K-H and Wilkens $M$ (to be published)

Essman U and Wilkans M 1964 Phys. Status Solidi 4 K 53

Eyre B L and Bullough R 1965 Philos. Mag. 1231

Eyre B L 1972 Defects in refractory metals (eds) R de Batist, J Nihoul and L Stals SCK/CEN Mol 311

Eyre B L, Loretto M H and Smallman R E 1977 Vacancies '76 (London: The Metals Society) 63

Jenkins M L, Katerban K H and Wilkens M 1976a Philos. Mag. 341141

Jenkins M L and Wilkens M 1976b Philos. Mag. 341155

Jenkins M L, Roller G, Katerban K H and Wilkens M 1982 J. Nucl. Mater. 108, 109603

Ruhle M 1967a Phys. Status Solidi 19263

Ruhle M 1967b Phys. Status Solidi 19279

Ruhle M 1971 on Radiation induced voids in metals, Proc. Int. Conf, Albany, New York 
Wilkens M 1970 Vacancies and interstitials in metals (eds) A Seeger, D Schumacher, W Schilling and J Diehl (Amsterdam: North Holland) 485

Wilkens M 1973 Applications of ion beams to metals (eds.) S T Picraux, E P Eer- Nisse and F L Vook (New York: Plenum Press) 441

Wilkens M 1978 Modern diffraction and imaging techniques (eds) S Amelinckx, R Gevers and J Van Landuyt (Amsterdam: North Holland Publ. Co.) 